\title{
The yeast SAS (something about silencing) protein complex contains a MYST-type putative acetyltransferase and functions with chromatin assembly factor ASF1
}

\author{
Shigehiro Osada, ${ }^{1,2}$ Ann Sutton, ${ }^{3}$ Nemone Muster, ${ }^{4}$ Christine E. Brown, ${ }^{1}$ John R. Yates III, ${ }^{4}$ \\ Rolf Sternglanz, ${ }^{3}$ and Jerry L. Workman ${ }^{1,5}$ \\ ${ }^{1}$ Howard Hughes Medical Institute (HHMI), Department of Biochemistry and Molecular Biology, The Pennsylvania State \\ University, University Park, Pennsylvania 16802-4500, USA; ${ }^{2}$ Laboratory of Environmental Biochemistry, Graduate School \\ of Pharmaceutical Sciences, Osaka University, Suita, Osaka 565-0871, Japan; ${ }^{3}$ Department of Biochemistry and Cell Biology, \\ State University of New York, Stony Brook, New York 11794-5215, USA; and ${ }^{4}$ Department of Cell Biology, The Scripps \\ Research Institute, La Jolla, California 92037, USA
}

It is well established that acetylation of histone and nonhistone proteins is intimately linked to transcriptional activation. However, loss of acetyltransferase activity has also been shown to cause silencing defects, implicating acetylation in gene silencing. The something about silencing (Sas) 2 protein of

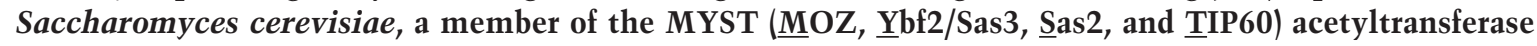
family, promotes silencing at $H M L$ and telomeres. Here we identify a $\sim 450-\mathrm{kD}$ SAS complex containing Sas $2 \mathrm{p}$, Sas $4 p$, and the tf $2 \mathrm{f}-$ related Sas 5 protein. Mutations in the conserved acetyl-CoA binding motif of Sas $2 \mathrm{p}$ are shown to disrupt the ability of Sas2p to mediate the silencing at $H M L$ and telomeres, providing evidence for an important role for the acetyltransferase activity of the SAS complex in silencing. Furthermore, the SAS complex is found to interact with chromatin assembly factor Asf1p, and asf1 mutants show silencing defects similar to mutants in the SAS complex. Thus, ASF1-dependent chromatin assembly may mediate the role of the SAS complex in silencing.

[Key Words: SAS; silencing; MYST; acetyl transferase; ASF1; chromatin assembly factor]

Received April 27, 2001; revised version accepted July 24, 2001.

Transcriptional silencing is a form of position-dependent and gene-independent transcriptional repression in which chromatin structure is altered over large regions of the genome. In Saccharomyces cerevisiae, silenced loci include the $H M L$ and $H M R$ mating-type loci, the telomeres, and genes within the ribosomal DNA. Many gene products have been identified that contribute to silencing (Sherman and Pillus 1997; Lustig 1998). Silent information regulator (Sir) proteins 1-4 are well-studied factors that play important functions in transcriptional silencing (Cockell et al. 1998). Sir2p, Sir3p, and Sir4p are components of a multiprotein complex, and mutations in any of these genes result in the complete derepression of the silent mating and telomere loci. In contrast to mutations in SIR2, SIR3, or SIR4, mutations in SIR1 only lead to a partial derepression of the silent-mating

${ }^{5}$ Corresponding author.

E-MAIL jlw10@psu.edu; FAX (814) 863-0099.

Article and publication are at http://www.genesdev.org/cgi/doi/10.1101/ gad.907201. loci. This is because in sir1 mutants, there is an epigenetic phenomenon in which some cells have fully silenced $H M$ loci, whereas others have fully expressed loci (Pillus and Rine 1989). Telomeric silencing, however, appears to be independent of Sirlp (Aparicio et al. 1991).

In a yeast genetic screen conducted to identify enhancers of sir1 epigenetic silencing defects, something about silencing (SAS) 2 was identified (Reifsnyder et al. 1996). The genetic experiments showed that $S A S 2$ has opposite regulatory effects, depending on the silenced locus. Sas2p promotes silencing at $H M L$ and telomeres but weakens it at an $H M R$ locus with mutations in $H M R-E$ silencer elements (Reifsnyder et al. 1996; EhrenhoferMurray et al. 1997). Recently, the SAS4 and SAS5 genes were also isolated as negative regulators of silencing at $H M R$-containing defective $H M R-E$ silencer elements (Xu et al. 1999a,b). SAS4 and SAS5 were also found to be positive regulators of silencing at $H M L$ and telomeres, and similar to $S A S 2$, each gene interacts genetically with SIR1 in silencing at HML. Strains containing null alleles of all combinations of sas 2 , sas 4 , and sas 5 were no more 
defective for $H M L$ silencing than any of the single deletion strains (Xu et al. 1999b). These genetic experiments suggest that SAS2, SAS4, and SAS5 function in the same genetic pathway.

Sas2p is a member of the MYST $(\underline{M O Z}, \underline{Y} b f 2 / S a s 3$, Sas2, and TIP60) family of acetyltransferases. MYST-related proteins have been identified from yeast to humans and include the following: human MOZ (Borrow et al. 1996), MORF (Champagne et al. 1999), TIP60 (Yamamoto and Horikoshi 1997), and HBO1 (Iizuka and Stillman 1999); Drosophila MOF (Smith et al. 2000); and yeast Sas2p (Reifsnyder et al. 1996), Sas3p (Takechi and Nakayama 1999; John et al. 2000), and Esalp (Smith et al. 1998). These MYST-related proteins show a high degree of sequence conservation in the acetyl-coenzyme A (acetyl-CoA) binding and zinc finger regions. Most MYST proteins have been shown to possess histone acetyltransferase (HAT) activity (Sterner and Berger 2000). Substrate specificity of the MYST acetyltransferases has also been investigated. For instance, in addition to HAT activity, TIP60 possesses autoacetylation activity (Creaven et al. 1999). Though Sas2p and MOZ contain the highly conserved acetyl-CoA binding motif, the histone acetylation activity and substrate specificity of these proteins have not been identified.

Many acetyltransferases are components of large multiprotein complexes in which associated subunits are often required for specific HAT activity and function in transcription (Howe et al. 1999; Brown et al. 2000). The complexes containing Sas3p, Esalp, dMOF, and hTIP60 have been purified and characterized (Allard et al. 1999; Ikura et al. 2000; John et al. 2000; Smith et al. 2000). The human TIP60 complex contains additional subunits possessing ATPase, DNA helicase, and DNA-binding activities (Ikura et al. 2000). Sas3p is the catalytic subunit of the HAT complex NuA3, which also contains the TBPassociated factor $\mathrm{TAF}_{\mathrm{II}} 30$. Sas3p mediates the interaction between NuA3 and Spt16p, a component of the yeast CP complex (Cdc68/Pob3) that functions in transcription elongation and DNA replication (John et al. 2000). NuA4 is 1.3 MD in size, and five Esalp-interacting subunits have been identified (Allard et al. 1999; Eisen et al. 2000; Galarneau et al. 2000). Characterization of these subunits provides us with important informa- tion about the role of HAT complexes in gene expression.

Histone acetylation is important for the regulation of gene silencing. Deletion of GCN5 enhances telomere silencing (Sun and Hampsey 1999). In contrast, deletion of $S A S 2$ leads to derepression of $H M L$ and a telomere proximal reporter gene (Reifsnyder et al. 1996). Mutations in HAT1, which encodes a subunit of a type B HAT, weaken telomeric silencing in the presence of certain histone tail mutations (Kelly et al. 2000). Furthermore, loss of the Nat1p-Ard1p cytoplasmic N-terminal acetyltransferase leads to silencing defects (Mullen et al. 1989). The significance of acetylation of histones and other proteins relevant to silencing remains unclear. Although SAS2 has been genetically associated with silencing, it is not known how the specific aspects of SAS2-mediated silencing are conferred. Here we identify and characterize the native Sas $2 p$ complex and investigate the effect of mutations in the Sas2p acetyl-CoA binding site on silencing in vivo. We also show that the Sas2 complex interacts with chromatin assembly factor Asflp, and deletion of both ASF1 and SIR1 causes derepression of HML. Further, we show that mutations in ASF1, like those in $S A S$ genes, improve silencing at mutated $H M R$ loci.

\section{Results}

The native Sas2 complex is $\sim 450 \mathrm{kD}$ in size

To gain further insight into the function of Sas2p, we sought to investigate whether it was a component of a multiprotein complex. To determine the molecular size of the native Sas2p-containing complex, Sas2p was tagged at its $\mathrm{C}$ terminus with the 13-Myc epitope by homologous recombination at the SAS2 chromosomal locus. Whole cell extracts were prepared from a strain expressing Sas2p-Myc (YJW265) and separated by Superose 6 size exclusion chromatography. Fractions were monitored for the presence of Sas2p-Myc by Western blot analysis. Only one protein peak was detected, which eluted in fractions 26 to 30 and corresponded to a molecular mass of $\sim 450 \mathrm{kD}$ (Fig. 1, upper panel). To confirm these results, two other strains that expressed either a C-terminal 3-HA epitope tagged Sas2p (YJW214) or an
Figure 1. Detection of a 450-kD Sas2p-containing complex. Yeast extracts were separated by Superose 6 size exclusion chromatography, and fractions were analyzed for Sas2 $p$ by Western blot analysis (anti-Myc or anti-HA monoclonal antibodies). (Top panel), Superose 6-separated whole cell extracts prepared from strain YJW265 expressing Sas2p-Myc. (Middle panel) Whole cell extracts prepared from strain YJW214, expressing Sas2pHA. The Sas $2 p-H A$ complexes were enriched by Mono Q anion-exchange chromatography before separation by Superose 6. (Bottom panel) Superose 6-separated whole cell extracts prepared from strain YJW213 expressing the GAL1-regulated HA-Sas $2 p$. Arrows mark peak fractions for molecular weight standard proteins.

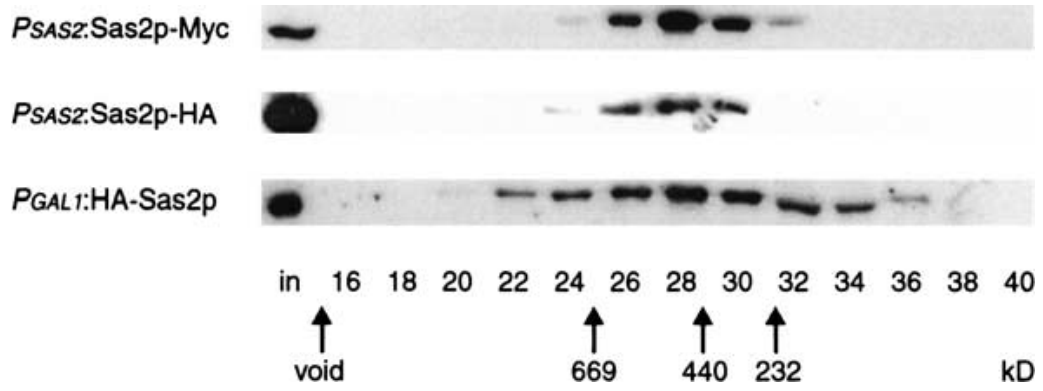


N-terminal 3-HA tagged Sas2p (YJW213) regulated by the GAL1 promoter were constructed. The Sas2p-HA complexes were enriched by Mono Q anion-exchange before separation by Superose 6 to improve detection of the native complex with the HA-antibody. Again, the Sas $2 \mathrm{p}-$ HA signal eluted in fractions 26 to 30 as observed for Sas2p-Myc (Fig. 1, middle panel). Similarly, when extracts prepared from the $G A L$-regulated HA-Sas2 strain were separated by Superose 6, the HA-Sas $2 \mathrm{p}$ also peaked in fractions 26 to 30 (Fig. 1, lower panel). These results indicate that the type and the position of the epitope tags do not affect the size of the Sas2 complex. Although the HA-Sas2p signal was spread out, this was likely caused by the overproduction of Sas $2 p$ by the strong GAL1 promoter. The results from the three chromosomally integrated Sas2p-tagged strains indicate that the size of the native Sas 2 complex is $\sim 450 \mathrm{kD}$.

\section{Sas $4 p$ and Sas5p are components of the Sas2 complex}

Genetic experiments have shown that SAS2, SAS4, and SAS5 are positive regulators of silencing at $H M L$ and telomeres and that the role of these $S A S$ genes in silencing of $H M L$ is not redundant (Xu et al. 1999b). These results suggest that Sas4p and Sas5p function in the same pathway as Sas2p. To investigate the size of the Sas4 and Sas5 complexes, C-terminal 13-Myc-tagged strains were generated (YJW228 and YJW229). Separation of whole cell extracts from the Sas4-Myc and Sas5-Myc strains by gel filtration showed that the Sas4p- and Sas5p-containing complexes are similar in size to the Sas2 complex (Fig. 2A). This raises the possibility that Sas2p, Sas4p, and Sas5p may in fact be components of the same complex. Accordingly, deletion of either SAS4 or SAS5 might alter the size of the Sas 2 complex. To examine this, whole cell extracts from wild type and sas $4 \Delta$, sas $5 \Delta$, and sas $4 \Delta$ sas $5 \Delta$ strains expressing Sas 2 p-
Myc were fractionated by Superdex 200 size exclusion chromatography (Fig. 2B). The Sas2p-Myc signal from the three deletion strains was more spread out and ran at significantly lower apparent molecular weights, although the Sas2 complex was present in all of the strains. The peak fractions of the Sas $2 \mathrm{p}-\mathrm{Myc}$ were shifted from fraction 25 to fractions 27,26 , and 27 by the deletion of the SAS4, SAS5, and SAS4/SAS5 genes, respectively. These results show that mutations in SAS4 and SAS5 alter the size or conformation of the Sas 2 complex and suggest that Sas $4 \mathrm{p}$ and Sas5p are components of the Sas2 complex.

To confirm the presence of Sas4p and Sas5p in the Sas 2 complex, double-tagged yeast strains containing $G A L$ regulated HA-Sas2p and Sas4p-Myc or Sas5p-Myc were generated (YJW230 and YJW231). Coimmunoprecipitation experiments were performed with whole cell extracts and peak Superose 6 fractions from the doubletagged strains grown in dextrose or galactose medium. Sas2p was immunoprecipitated with HA-antibody, and coprecipitation of Sas $4 \mathrm{p}$ and Sas5 $\mathrm{p}$ was monitored by anti-Myc Western analysis. As shown in Figure 3, Sas4p and Sas5p coprecipitated with Sas $2 \mathrm{p}$ from both the whole cell extracts and the peak fractions of Superose 6 only when HA-Sas2 expression was induced with galactose. Control experiments performed with extracts and Superose 6 fractions prepared from strains grown in dextrose medium show that efficient coprecipitation of Sas $4 p$ and Sas5p was dependent on their interaction with Sas2p. The low level of Sas4p-Myc detected in the bead fraction when the strain was grown on dextrose is likely caused by leakiness of the GAL1 promoter. The cofractionation of Sas $2 p$ with Sas $4 p$ and Sas5p and the immunoprecipitation experiments indicate that Sas $4 p$ and Sas5p are indeed components of the Sas 2 complex. Thus, we named the Sas 2 complex, which also contains Sas $4 p$ and Sas5p, simply the SAS complex.
A
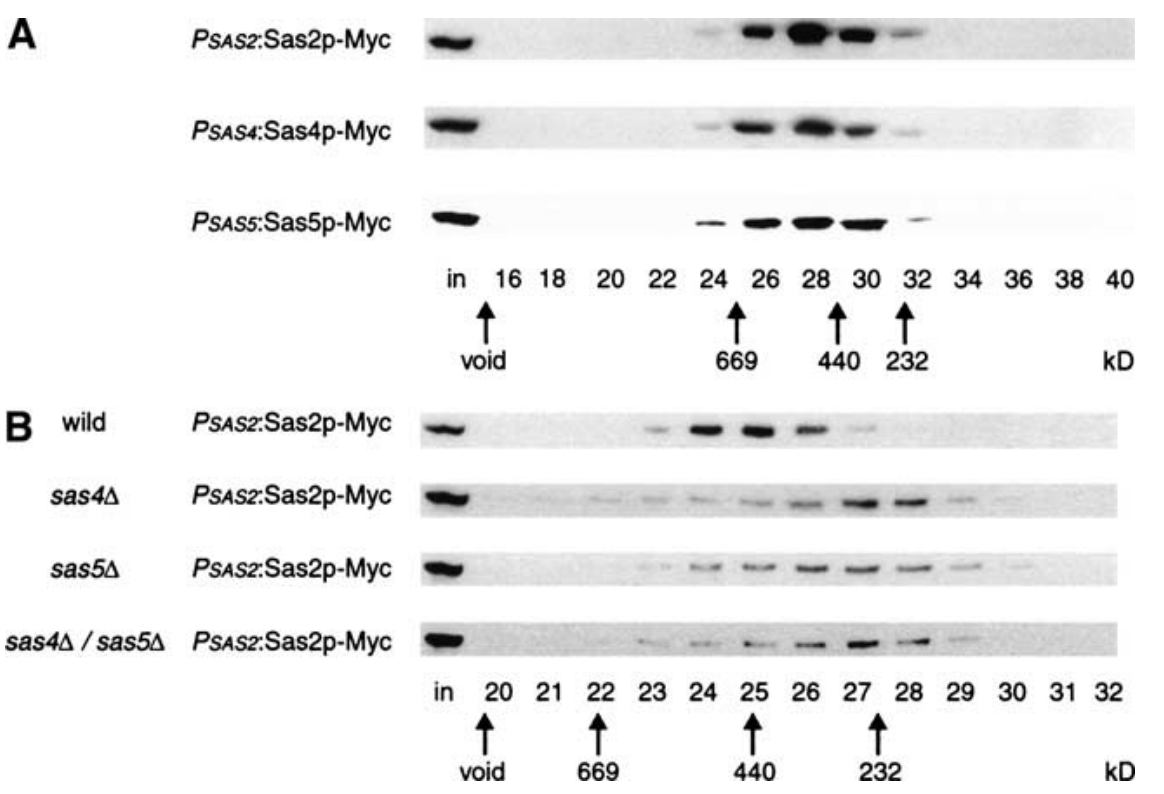

kD
Figure 2. Sas4p and Sas5p are components of the Sas2 complex. (A) Extracts were prepared from strains YJW265, YJW228, and YJW229 expressing the kD C-terminal Myc-tagged Sas2, Sas4, and Sas5 proteins, respectively. The sizes of the Sas2, Sas4, and Sas5 complexes were determined by analysis of elution profiles from Superose 6. (B) Comparison of the Sas2p-Myc elution profiles from Superdex 200 size exclusion chromatography following fractionation of whole cell extracts prepared from wild type (YJW265) and

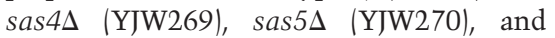

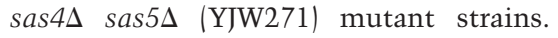
Shown are Western blots of column fractions probed with Myc-antibodies. 
Figure 3. Sas $4 p$ and Sas5p coimmunoprecipitate with Sas2p. Coimmunoprecipitation experiments were performed with both whole cell extracts (WCE) and Superose 6 fraction 28 prepared from doubletagged strains HA-Sas2p, Sas4p-Myc (YJW230) and HA-Sas2p, Sas5-Myc (YJW231; indicated at the bottom of the panel). Protein fractions were incubated with HA-antibodies, and $10 \%$ of input (in), $10 \%$ of supernatant (sup), and bead (be) fractions from the immunoprecipitates were probed for Myc-tagged proteins.
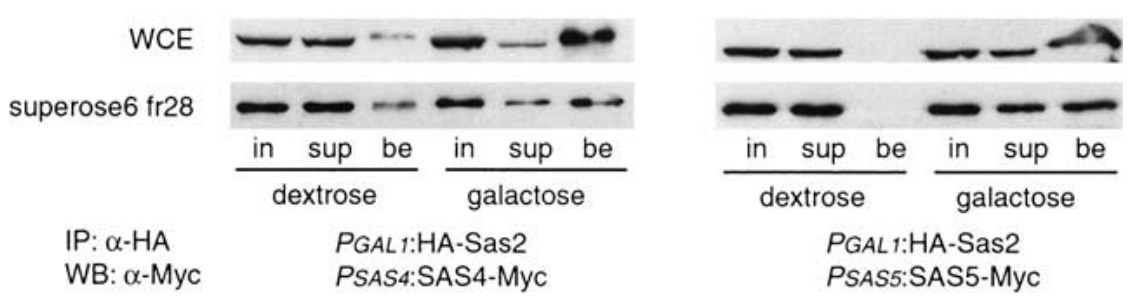

\section{Purification and characterization of the SAS complex}

To further characterize the SAS complex, it was purified to homogeneity. For this purpose, a double-tagged yeast strain harboring chromosomally integrated Sas4p-Myc and a plasmid expressing Sas $2 \mathrm{p}$ tagged with an N-terminal $6 \mathrm{xHis}$ and a C-terminal Flag and regulated by the GAL10 promoter was used (YJW276). This SAS2 expression plasmid was also used for the mating assays and could fully complement the sas2 deletion (see Fig. 5B). The SAS complex was purified on the basis of the Sas4pMyc signal, using the anti-Myc Western blot analysis as outlined in Figure 4A. The Flag affinity-purified SAS complex was fractionated by Superdex 200, and each fraction was analyzed by silver stain and Western blot analysis (Fig. 4B). The major Sas2p peak (fraction 23, 24) corresponds to a molecular mass of $\sim 450 \mathrm{kD}$, similar to the complex characterized in Figures 1 and 2. Western blot analysis revealed that Sas4p-Myc (p100) was only found in the $450-\mathrm{kD}$ Sas2 complex (Fig. 4B, middle panel). A significant portion of Sas $2 p$ was also detected at higher molecular weights (e.g., fraction 20). Whether this is a unique complex containing Sas2p or an artifact of overexpression of Sas2p is under investigation. As a control, we performed mock purification from whole cell extracts prepared from the wild-type parental strain containing an empty expression vector (YJW243). No protein bands were detected in the Flag eluate from the control strain by silver staining (data not shown).

Three major bands (p100, p72, and p30) cofractionated with the Sas2p (p40) peak fraction. To identify the coeluting proteins, the $\mathrm{p} 100, \mathrm{p} 72, \mathrm{p} 40$, and $\mathrm{p} 30$ bands were excised and analyzed by mass spectrometry. Twelve, four, and three peptides obtained from p100, p40, and p30 corresponded to the Sas $4 p$, Sas $2 p$, and Sas5p, respectively (Fig. 4C). This finding confirmed that Sas2p, Sas $4 p$, and Sas5p co-associate in a unique and distinct complex. Thirteen peptide sequences from p72 correspond to six different yeast proteins. Experiments are in progress to confirm which, if any, of these proteins are bona fide subunits of the SAS complex.

The conserved acetyl-CoA binding motif of Sas $2 p$ is required for $H M L$ and telomere silencing

The yeast Sas2, Sas3, and Esal proteins all show a significant degree of conservation in the MYST-related ace-

tyl-CoA binding site (Fig. 5A). However, for some MYST proteins (including Sas2p), HAT activity has not been described yet. To test for Sas2p HAT activity, we performed HAT assays with the purified SAS complex. Partially and highly purified fractions of the SAS complex failed to acetylate free or nucleosomal histones in vitro (data not shown). Recombinant Sas2p could not be assayed because it was insoluble. It is possible that Sas $2 p$ might require other cofactors to acetylate histones or that it acetylates nonhistone proteins. Either possibility would suggest that the highly conserved acetyl-CoA binding motif in Sas2p should be essential for its function. Mutations within the predicted acetyl-CoA binding pocket in Sas3p (M1 429GYG to AAA; M2 426QR to AA) have been shown to greatly reduce HAT activity of the NuA3 complex (John et al. 2000). By comparison, a Sas3p (M3 434LM to AA) mutation in the hydrophobic pocket somewhat distal to the acetyl-CoA binding site did not affect NuA3 HAT activity (John et al. 2000). To investigate the role of the Sas2p putative acetyl-CoA binding site in vivo, we mutated conserved amino acids that are predicted to be important for acetyl-CoA binding and examined their effect on silencing at the silent matingtype loci and at a telomere. For this purpose, we constructed point mutants in $S A S 2$, which corresponded to SAS3 mutants M1, M2, and M3 (Fig. 5A).

The $S A S 2$ gene was originally isolated as an enhancer of the epigenetic silencing defects of yeast sir 1 mutants. SAS2 also contributes to silencing at the telomeres (Reifsnyder et al. 1996). The combined deletion of sir1 and sas2 genes causes a much more severe silencing defect at $H M L$ than does the deletion of sir 1 alone. To investigate the relevance of the Sas2p putative acetyl-CoA binding site on HML silencing, wild-type and mutated $S A S 2$ alleles were transformed into the sir1 sas2 double-mutant strain (Fig. 5B). We constructed three types of expression vectors. Two of them contain the native SAS2 promoter on either CEN or $2 \mu$ plasmids, and the other is regulated by the GAL10 promoter. As judged by mating assays, the sas2-M3 mutant restored $H M L$ silencing to wild-type levels when overexpressed and to intermediate levels when expressed from a $C E N$ plasmid (Fig. 5B). However, mutant strains carrying the sas2-M1 mutation could no longer restore the mating-type silencing (Fig. 5B). Thus, residues within the acetyl-CoA binding domain (M1), not distal (M3), are absolutely required for the SAS2 silencing function. This result indicates that the con- 
SAS complex interacts with ASF1

A

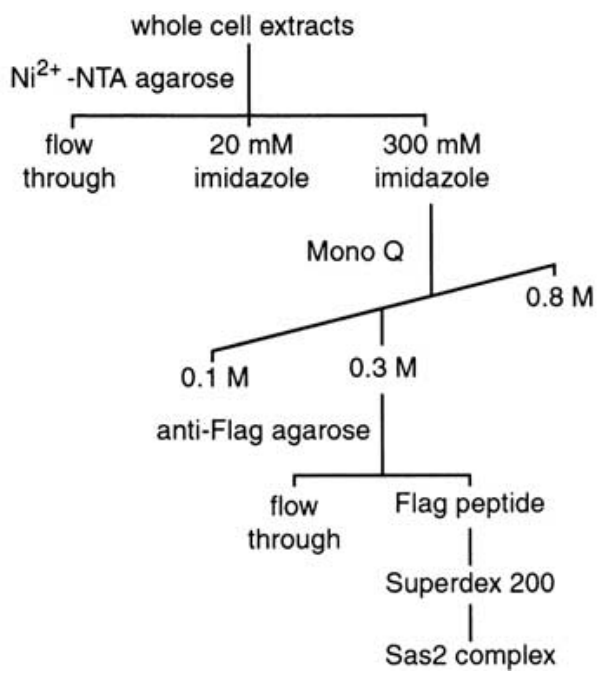

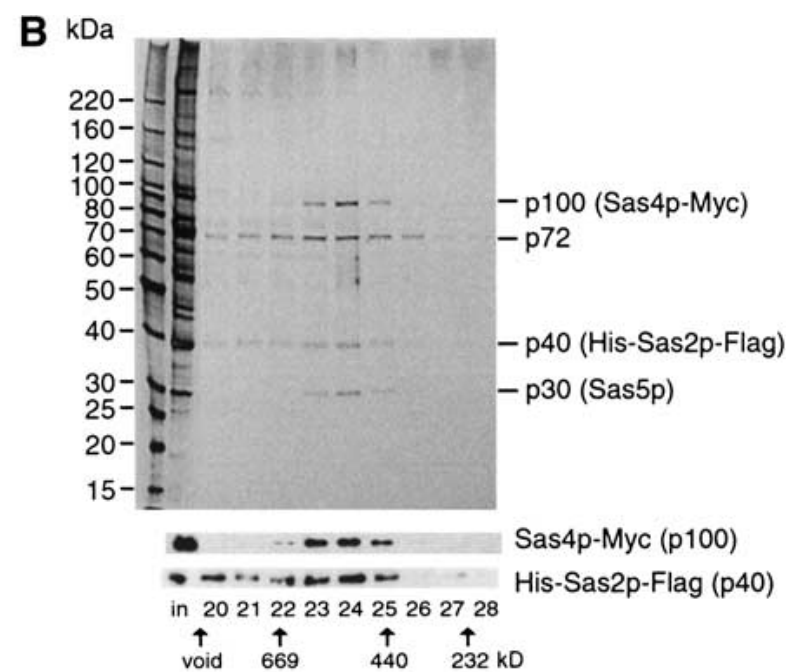

\section{Sas2p (p40)}

4 SLSQSLTATTQK 15

4 SLSQSLTATTQKLK 17

61 FSTWYGSAVYFDPETK 76

61 FSTWYGSAVYFDPETKR 77

\author{
Sas4p (p100) \\ 24 NSHGEFEKFDFDTEEYEINPK 44 \\ 65 SCFTVDEHVDETR 77 \\ 85 SPFMSNVDDEIKK 97 \\ 105 MTLEIEHHELSQNIR 119 \\ 120 KPTDDLLPDSTYQPY 134 \\ 120 KPTDDLLPDSTYQPYHK 136 \\ 145 MIQSDIVNGENEADR 159 \\ 182 INDPTDENEMETK 194 \\ 352 EVIPLTMEVEPEVIR 366 \\ 401 NNAASISPTLSEK 413 \\ 414 APLGSISSCTASQISQRAPLGSISSCTASQISQR 430 \\ $\triangle 66$ TNSGINILPVR 476
}

\section{Sas5p (p30) \\ 13 TQQVIIPEQNIR 24 \\ 161 SLALIDEDMMTDVVQMILNDPAVQRSLALIDEDMMTDVVQMILNDPAVQR 185 \\ 228 TNFGSDAIHKDEPVK 242}

Figure 4. Purification of the SAS complex. (A) Schematic representation of the chromatographic steps applied for purification of the SAS complex. $(B)$ The SAS complex was purified from YJW276. (Top) A silver-stained gel of fractions following Superdex 200 chromatography. (Bottom) Western blot of Superdex 200 fractions. Specific bands in the smaller Sas2 complex are indicated by their molecular weight. Sas4p-Myc and His-Sas2p-Flag were detected by Western blot analysis with antiMyc and anti-Flag antibodies. (C) Peptide sequences obtained from mass spectrometry analysis are shown. Numbers at the left and right of the sequence indicate the position in each protein, respectively. served acetyl-CoA binding motif in Sas2p is necessary for the $H M L$ silencing in vivo and strongly suggests that Sas $2 \mathrm{p}$ possesses acetyltransferase activity in vivo.

Unexpectedly, the sas2-M2 mutant was also able to restore $H M L$ silencing (Fig. 5B). By analogy to the Esalp crystal structure, residues corresponding to M1 and M2 interact with acetyl-CoA. The crystal structure of Esalp/ $\mathrm{CoA}$ indicates that the M1 mutation, which includes two Gly to Ala mutations, would likely disrupt the conformation of the $\alpha 3$ helix and loop observed to interact with acetyl-CoA (Yan et al. 2000). In contrast, the M2 mutation residues are predicted to make modest interactions with acetyl-CoA. Removing side-chains of Esalp Gln312 and Arg313 would be expected to weaken the Esalp/CoA interactions only slightly. We therefore pre- dict that the mutant Sas2-M2 might retain weak acetylation activity that is sufficient for restoring silencing. This evidence is also supported by telomere silencing experiments (described below).

It was formally possible that the sas2-M1 mutant did not rescue silencing because of a lack of protein expression or a failure to become incorporated into the SAS complex. To rule this out, the SAS complex was partially purified from strains (YJW275, 278-280) carrying wildtype and mutant His-Sas2p-Flag expression constructs. Anti-Flag Western blot of fractions from anion exchange and size exclusion columns indicated that the abundance and the size of the mutant SAS complexes were unchanged (data not shown).

To investigate the effect of the mutations in the con- 
A

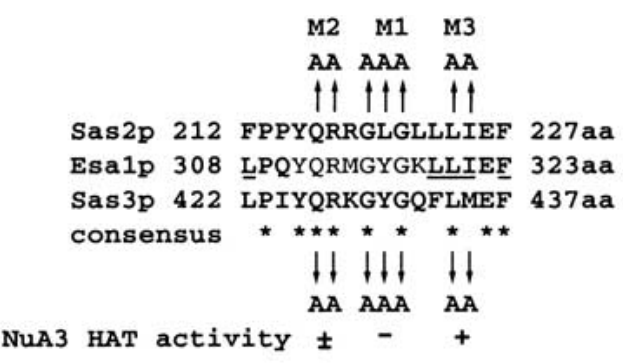

B

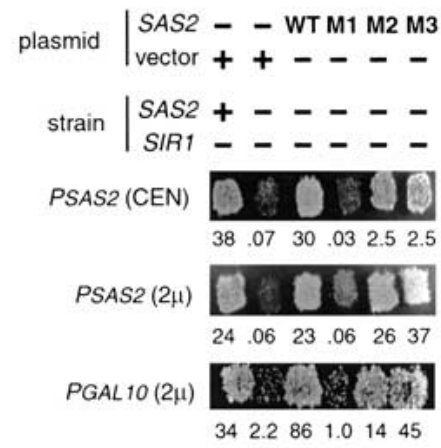

C

$\begin{array}{clll}\frac{\text { strain }}{\text { SAS2 SIR1 }} & & \text { vlasmid } \\ +\mathbf{+} & \mathbf{+} & \mathbf{+}\end{array}$

$\begin{array}{cccc}+ & + & + & - \\ - & + & + & - \\ - & + & - & \mathrm{WT} \\ - & + & - & \mathrm{M} 1 \\ - & + & - & \mathrm{M} 2 \\ - & + & - & \mathrm{M} 3\end{array}$
5-FOA

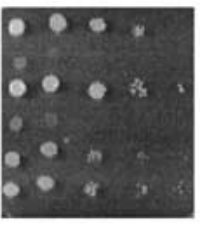

$\begin{array}{llll}+ & - & + & - \\ - & - & + & - \\ - & - & - & \text { WT } \\ - & - & - & \text { M1 } \\ - & - & - & \text { M2 } \\ - & - & - & \text { M3 }\end{array}$

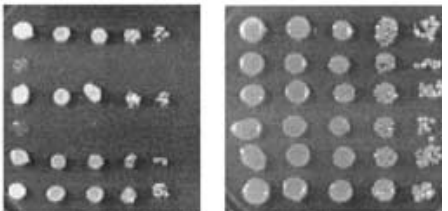

Figure 5. The conserved acetyl-CoA binding motif of Sas2p is required for $H M L$ and telomeric silencing. (A) Sequence comparison of acetyl-CoA binding motifs of Sas2p, Sas3p, and Esalp. The positions of the mutated amino acid residues are shown. The residues of Esalp involved in acetyl-CoA binding are indicated in gray, and underlined amino acids represent buried residues (Yan et al. 2000). (B) The effect of the SAS2 acetyl-CoA binding site mutations on silencing at $H M L$ was assayed by measuring mating efficiencies. Strains expressing wild-type and mutant alleles of $S A S 2$ from CEN-based plasmids containing the SAS2 promoter (YJW348, YJW350, YJW352, YJW353, YJW354, and YJW355; ordered from left to right), $2 \mu$-based plasmids also containing the SAS2 promoter (YJW349, YJW351, YJW356, YJW357, YJW358, and YJW359|, and 2 $\mu$-based plasmids regulated by the GAL10 promoter (YJW281, YJW282, YJW283, YJW284, YJW285, and YJW286) were patched onto plates lacking uracil and replica plated onto minimal medium spread with a lawn of MAT $\alpha$ cells. Quantitative mating analyses of the same strains are expressed as a percentage of diploids formed per viable cells. $(C)$ To evaluate the expression of a telomere-proximal URA3 gene, serial dilutions of saturated cultures were inoculated onto plates lacking leucine in the presence and absence of 5-FOA. Silencing was compared for the following strains (top to bottom): YJW324, YJW328, YJW330, YJW369, YJW370, YJW371, YJW326, YJW332, YJW334, YJW375, YJW376, and YJW377. served acetyl-CoA binding site on telomeric silencing, plasmids containing wild-type and mutated SAS2 alleles were transformed into a sas2 deletion strain containing the $U R A 3$ gene inserted into the $A D H 4$ locus adjacent to an artificial telomere. The silencing efficiency was measured by 5-fluoroorotic acid (5-FOA) sensitivity. 5-FOA is toxic to $\mathrm{Ura}^{+}$cells. Lack of telomeric silencing, and thus expression of the URA3 gene, results in sensitivity to 5-FOA, whereas strains that repress URA3 transcription are resistant to 5-FOA. The wild-type strain containing the empty expression vector grew on 5-FOA plates because the URA3 gene was repressed. In contrast, the sas2 deletion strain was sensitive to 5-FOA, in agreement with a previous report by Reifsnyder et al. (1996). Although the plasmid containing the wild type and the innocuous mutant sas2-M3 gene fully complemented the deletion of SAS2, the sas2-M1 plasmid showed no detectable complementation activity and behaved like the empty vector. These results were obtained from both SIR1 wild-type and sir1 deletion strains. Interestingly, the mutant sas2-M2 showed only partial restoration of silencing in SIR1 cells but restored wild-type levels of silencing in sir1 deletion strains. This is surprising because Sirlp does not play a major role in the silencing of telomeric reporter genes (Aparicio et al. 1991). Perhaps deletion of SIR1 releases Sir2p, Sir3p, and Sir4p from the $H M$ loci and thus improves telomeric silencing.

Taken together, the mating-type loci and telomere silencing experiments indicate that the conserved acetylCoA binding site in Sas2p is required for HML and telomeric silencing. The severity of the effect of different mutations correlates with their predicted effect on acetyl-CoA binding. These results suggest that the acetyltransferase activity of Sas2p is necessary for its function in the silencing in vivo.

\section{Asf1p interacts with the SAS complex}

Sas4p (amino acids 339-481) was found in a two-hybrid screen with chromatin assembly factor Asf1 (anti-silencing function 1; Sutton et al. 2001). ASF1 was originally identified as a gene that derepresses transcriptional silencing when overexpressed (Le et al. 1997; Singer et al. 1998). Drosophila ASF1 has been shown to be a component of the replication-dependent chromatin assembly factor (RCAF) complex (Tyler et al. 1999). Human Asflp associates with acetylated histone $\mathrm{H} 3$ and $\mathrm{H} 4$, functioning as a histone chaperone for chromatin assembly in vitro (Munakata et al. 2000). To determine whether Asflp interacts with Sas4p in vitro, GST pull-down assays were performed (Fig. 6A). ${ }^{35}$ S-labeled Sas $4 p$ interacted with GST-Asf1p but not with GST alone. By comparison, ${ }^{35}$ S-labeled Sas2p and Sas5p did not bind to GST-Asf1p. These results suggest that only Sas $4 p$ interacts with Asflp in the absence of the other two subunits. To provide evidence that Asflp interacts with Sas4p in vivo, immunoprecipitation experiments were performed (Fig. 6B). Whole cell extracts from strains expressing HAtagged or nontagged Asflp with Sas4-Myc protein were incubated with HA-antibody and precipitated. Immuno- 


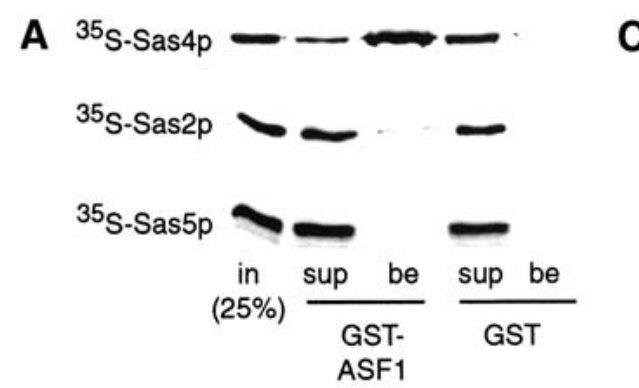

B
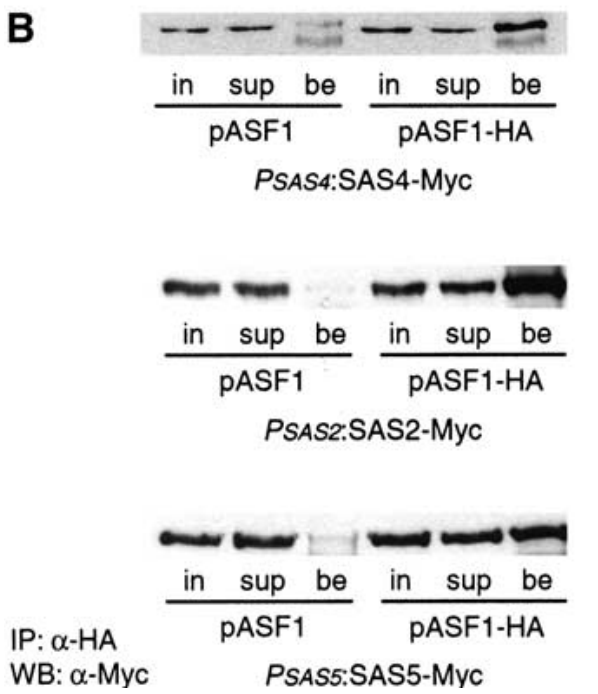

D
C

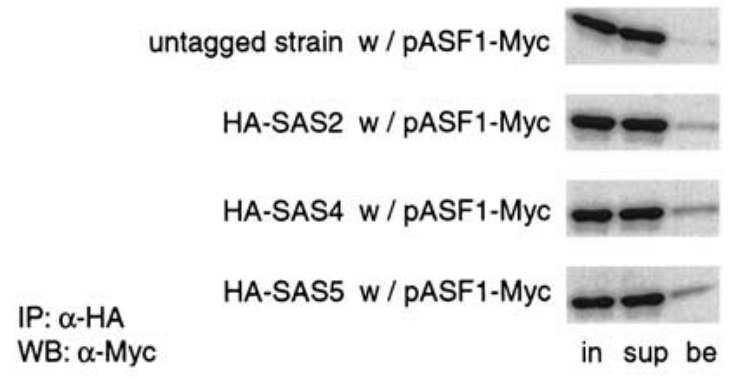

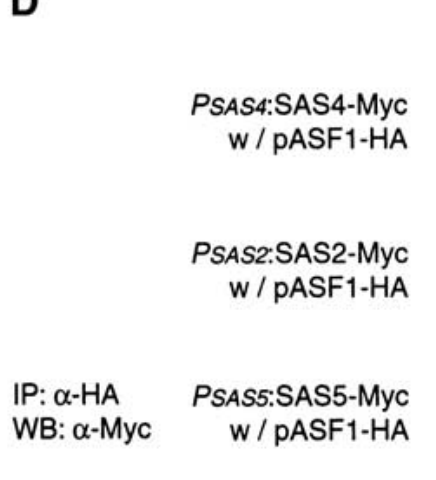

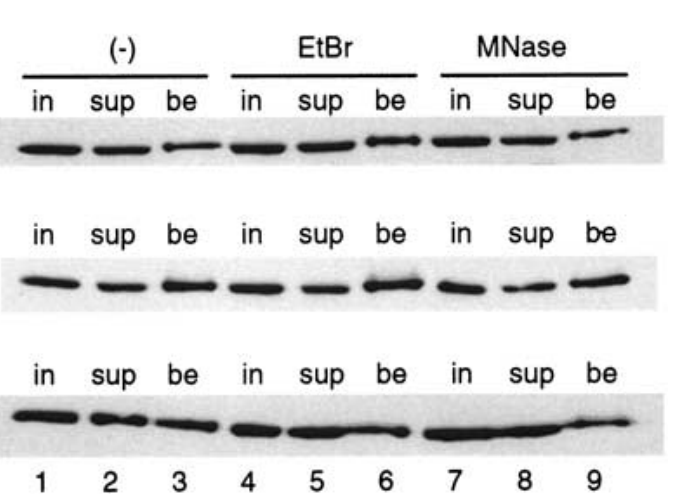

Figure 6. The SAS complex interacts with Asflp. (A) Individually expressed ${ }^{35}$ S-labeled Sas proteins were incubated with GST or GST-Asf1 fusion proteins coupled to glutathione Sepharose beads. Proteins that remained bound to the beads fraction (be) were subjected to SDS-PAGE. The amount of input (in) and supernatant (sup) are equivalent to $25 \%$ of the reaction in the assay. (B) Coimmunoprecipitation experiments were performed with whole cell extracts prepared from the double-tagged strains expressing the Sas4p-Myc (top, YJW416 and YJW417), Sas2p-Myc (middle, YJW414 and YJW415), Sas5p-Myc (bottom, YJW418 and YJW419), and Asflp or Asf1p-HA (indicated at the bottom of the panel). Whole cell extracts were incubated with anti-HA antibodies, and $0.5 \%$ of input (in), $0.5 \%$ of supernatant (sup), and bead (be) fractions from the immunoprecipitates were probed for the Myc-tagged proteins. (C) The HA-tagged Sas proteins coimmunoprecipitate Asflp. The Myc-tagged ASF1p expression plasmid was transformed into the untagged strain and the chromosomally integrated HA-tagged SAS2, SAS4, and SAS5 strains (YJW457, YJW458, YJW459, and YJW460). Results are as described in $B .(D)$ The interaction between the SAS complex and ASF1p is not mediated via DNA/chromatin in the extracts. Coimmunoprecipitation experiments were performed in untreated WCE (lanes 1-3), presence of ethidium bromide (EtBr) (lanes 4-6), or presence of MNase (lanes 7-9). Whole cell extracts were prepared from strains expressing the Sas4p-Myc (top, YJW417), Sas2p-Myc (middle, YJW415), Sas5p-Myc (bottom, YJW419). Results are as described in Figure 6B except that 1\% of input and supernatant was loaded.

precipitated Sas4-Myc protein was only detected on the beads from the strain expressing HA-tagged Asflp, indicating that Asflp interacts with Sas4p at physiological levels. We have shown that Sas $4 p$ is a component of the SAS complex (Figs. 2-4). If Asf1p interacts with the SAS complex, Sas2p and Sas5p should also coprecipitate with Asflp-HA. Immunoprecipitation experiments using Asflp-HA and nontagged Asflp showed that Asflp-HA interacts with both the Sas2p-Myc and Sas5p-Myc (Fig. 6B). To provide further support for the Asf1-SAS complex interaction, reciprocal immunoprecipitation experiments were performed (Fig. 6C). For these experiments, a plasmid expressing Asflp, containing a C-terminal Myc tag regulated by the native promoter, was transformed into strains harboring chromosomally integrated HASas2p, HA-Sas4p, and HA-Sas5p (YJW458, YJW459, and
YJW460). As a control, Asf1p-Myc expression plasmid was transformed into the untagged strain (YJW457). Extracts from strains expressing untagged Sas proteins or HA-tagged Sas proteins were precipitated with HA-antibody, and coprecipitation of Asflp was detected by antiMyc Western blot analysis. Consistent with our previous findings, all of HA-tagged Sas proteins coimmunoprecipitated Asflp-Myc. These results indicate that Asflp interacts with the native SAS complex in vivo. The fraction of Myc-tagged Asflp that coprecipitated with HASas proteins is less than the fraction of Myc-tagged Sas proteins that copreciptiated with Asf1-HA (Fig. 6B,C). This indicates that only a fraction of Asflp is associated with the SAS complex and is consistent with the fact that Asflp is also found in other complexes (e.g., with Rad53) that function in processes independent of silenc- 
ing (e.g., DNA repair; Le et al. 1997; Tyler et al. 1999; Emili et al. 2001; see below).

To rule out the possibility that the interaction between the SAS complex and ASF1 is mediated via DNA/ chromatin, extracts were treated before immunoprecipitation with either ethidium bromide $(\mathrm{EtBr})$ or micrococcal nuclease (MNase). These reagents disrupt DNAprotein interaction by either distorting DNA structure by intercalation (EtBr) or by degrading DNA (MNase). The efficiency of interaction between Asf1 and Sas proteins was not influenced by the addition of either EtBr or MNase (Fig. 6D), indicating that the interaction between the native SAS complex and Asflp is not mediated via DNA/chromatin.

The effect of ASF1, SIR1, and SAS2 disruption on sensitivity to mutagens

asf1 mutants show multiple phenotypes, including slow growth, a weak sensitivity to ultraviolet light, a sensitivity to the DNA alkylating reagent methyl methane sulfonate (MMS), and a sensitivity to hydroxyurea (HU), a chemical inhibitor of ribonucleotide reductase that blocks DNA replication by impairing dNTP synthesis (Le et al. 1997; Tyler et al. 1999; Emili et al. 2001). The functional interaction between ASF1 and RAD53 (DNA damage checkpoint protein gene) may play a role in the control of DNA repair (Emili et al. 2001; Hu et al. 2001). ASF1 also contributes to gene silencing. Overexpression of $A S F 1$ reduces silencing at mating-type loci, telomeres, and ribosomal DNA (Le et al. 1997; Singer et al. 1998). Though the asf1 mutant showed no significant defect in the silencing at mating-type loci and telomeres, deletion of ASF1 in combination with a null allele of CAC1 or $C A C 2$, which encode subunits of chromatin assembly factor 1 (CAF-1), weakens gene silencing (Tyler et al. 1999; Sutton et al. 2001).

To test for a role of the SAS complex and Sir1p in DNA repair, we examined the effects of disruption of ASF1 in combination with a null allele of SIR1 and/or SAS2 on sensitivity to mutagens (Fig. 7A). Although asf1 mutants grow slowly, no additional effects on growth by disruption of ASF1 in sas2 and/or sir1 deletion strains were observed. Mutation of SIR1 and/or SAS2 in both ASF1 and asf 1 strains did not enhance the sensitivity to DNA damaging agents or the DNA replication inhibitor. These results indicate that SIR1 and SAS2 are not involved in ASF1-mediated DNA repair and DNA replication and suggest that interaction between the SAS complex and Asflp is important for different ASF1 functions.

\section{asf1 mutants affect $H M L$ and $H M R$ silencing}

To investigate whether Asflp and the SAS complex function together, we tested the mating efficiencies of strains

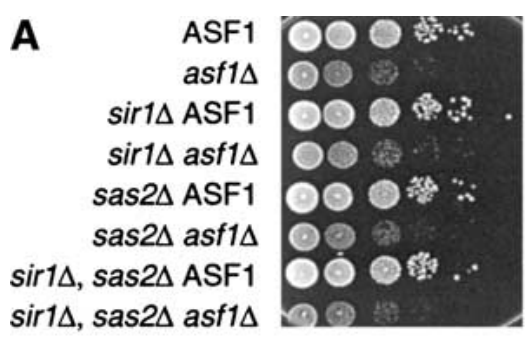

$(-)$

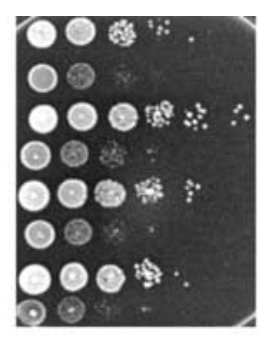

UV

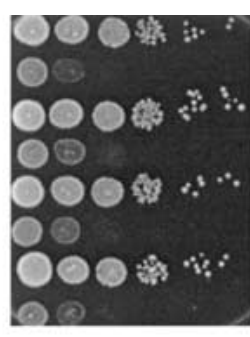

MMS

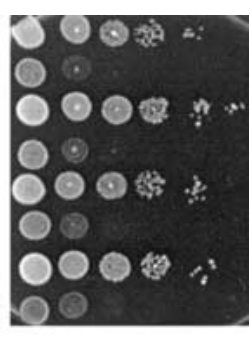

HU
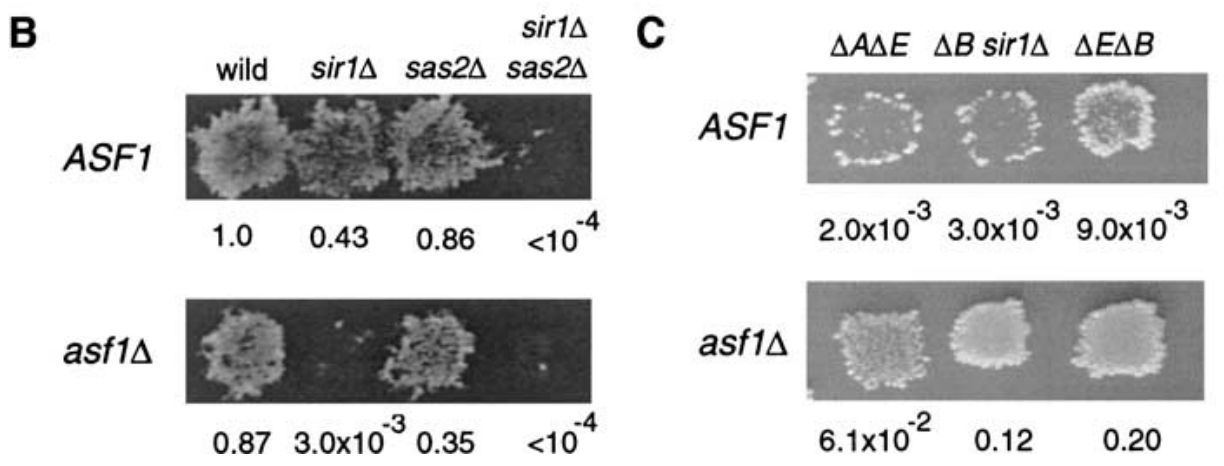

Figure 7. DNA damage sensitivity and mating efficiencies of asf1, sir1, and sas2 mutants. (A) Effects of disruption of ASF1 and SIR1 and/or SAS2 on sensitivity to mutagens. Serial dilutions of wild type and mutant strains were spotted and grown on complete media in the absence (-), or presence of ultraviolet radiation $\left(100 \mathrm{~J} / \mathrm{m}^{2}\right)$, MMS $(0.006 \%)$, or HU $(25 \mathrm{mM})$. Strains analyzed were W303-1a (wild

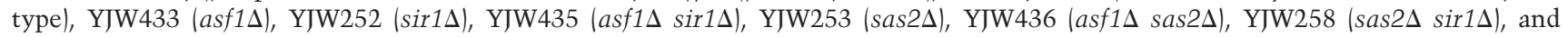
YJW437 (asf1 $1 \Delta$ sas2 2 sir1 $\Delta)$. (B) ASF1 contributes to silencing at $H M L$. Mating assays are shown with quantitative mating assay results

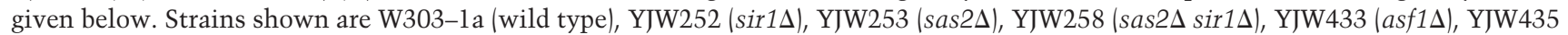

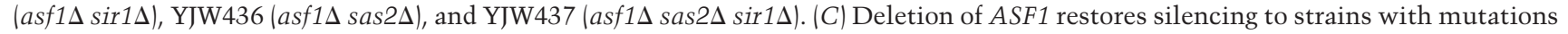
in $H M R$-E. Quantitative mating assay results are expressed relative to a value of 1.0 for wild-type W303-1b. Strains shown are YAB53 $(\Delta A \Delta E)$, YAB197 ( $\Delta B \operatorname{sir} 1 \Delta)$, YGM75 ( $\Delta E \Delta B)$, SY557 (asf1 $\Delta \Delta A \Delta E)$, SY558 (asf1 $\Delta \Delta B \operatorname{sir} 1 \Delta)$, and SY560 (asf1 $\Delta \Delta E \Delta B)$. 
harboring a null allele of $A S F 1$ in combination with null alleles of SIR1 and/or SAS2 (Fig. 7B). Similar to deletion of either SIR1 or SAS2, deletion of ASF1 results in a very slight reduction in silencing at $H M L$, as indicated by quantitative mating analysis. On the other hand, the mating ability of a asf1 sir 1 double mutant was three orders of magnitude less than that of wild type or single deleted strains and was almost the same as that of double sas2 sir1 or triple asf1 sas2 sir1 deletion strains. A strain containing null alleles of both ASF1 and SAS2 was only slightly more defective than strains individually deleted for these genes. These results suggest that both Asflp and the SAS complex function in a pathway that enhances the epigenetic silencing defects of sir 1 mutants.

In contrast to their effect on silencing at $H M L$, deletions of SAS2, SAS4, and SAS5 improve silencing at an $H M R$ locus that has mutations in E silencer elements (Reifsnyder et al. 1996; Ehrenhofer-Murray et al. 1997; Xu et al. 1999a). We tested the effect of deletion of ASF1 on $H M R$ silencing by measuring the mating ability of three $M A T \alpha$ strains with mutated $H M R$-E elements (Fig. 7C). In all three cases, loss of ASF1 resulted in a substantial improvement of mating, as has been previously reported for the $S A S$ genes.

\section{Discussion}

We identified and characterized a SAS complex that is $\sim 450 \mathrm{kD}$ in size and contains Sas2p, Sas4p, and Sas5p. There are several lines of evidence that lead us to this conclusion. First, size exclusion chromatography showed that complexes containing Sas4p and Sas5p are similar in size to the protein complex containing Sas $2 p$ (Fig. 2A). Second, deletion of SAS4 and/or SAS5 alters the size of the Sas 2 complex (Fig. 2B). Third, Sas $4 p$ and Sas5p coimmunoprecipitated with Sas2p (Fig. 3). Fourth, a highly purified Sas 2 complex contains Sas4p and Sas5p (Fig. 4B). Finally, the mass spectrometry data confirmed that Sas $4 p$ and Sas5p are components of the Sas 2 complex (Fig. 4C). The existence of Sas2p, Sas4p, and Sas5p in the same complex explains recent studies, which have showed that the SAS2, SAS4, and SAS5 have similar functions in silencing of $H M L$ (Xu et al. 1999b).

In addition to the characterization of the subunits of the SAS complex, we have shown that the conserved acetyl-CoA binding site in Sas2p is required for HML and telomeric silencing (Fig. 5). This strongly suggests that acetyltransferase activity of the SAS complex is critical for silencing in vivo. However, the substrate of the SAS complex is currently unknown. We could not detect any HAT activity in partially or highly purified preparations of the SAS complex using free or nucleosomal histones (data not shown). Although these are negative results, they do suggest that the substrate of Sas2 might be proteins other than histones. We have provided strong evidence that the acetyl-CoA binding domain is essential for the silencing function of Sas $2 p$ in vivo. The identification of the substrate of the SAS complex will enable us to address the silencing mechanism mediated by the Sas2-dependent acetylation.

\section{SAS complex links DNA replication and silencing to chromatin modification}

It has been shown that the restoration of silencing at the $H M R$ locus with a mutated silencer by a sas 2 mutant required Orc $2 p$ and Orc5p, which are subunits of the origin recognition complex (ORC; Ehrenhofer-Murray et al. 1997). ORC is an initiator complex for DNA replication but also affects transcriptional silencing (Dutta and Bell 1997). HBO1 (histone acetyltransferase binding to ORC) is a member of the MYST acetyltransferases family and has been identified as an interaction partner of the human ORC1 protein (Iizuka and Stillman 1999). $\mathrm{HBO} 1$ also interacted with the androgen receptor and a transcriptional repression domain of HBO1 was identified (Sharma et al. 2000). Genetic interactions between $S A S 2$ and ORC-encoding genes suggest that $\mathrm{HBO} 1$ might be functionally related to the Sas2 protein. Thus, the Sas2 complex might play a key role in linking DNA replication and gene silencing to chromatin modification.

\section{Sas5p is a tf2f domain-containing protein}

It remains unclear how SAS4 and SAS5 mediate SAS2dependent silencing. Sas $4 \mathrm{p}$ and Sas5p might be essential for the structural integrity of the SAS complex, because deletion of the SAS4 and/or SAS5 genes altered the size of the SAS complex. Sas4p has no obvious homology with other known proteins. A BLAST search (Altschul et al. 1997) reveals that an $\mathrm{N}$-terminal stretch of $\sim 100$ amino acids in Sas5p has homology with proteins that have been identified in S. cerevisiae, S. pombe, Caenorhabditis elegans, Drosophila, Arabidopsis, and humans. This domain is $\sim 60 \%$ homologous in all these proteins and is registered as the tf $2 \mathrm{f}$ domain in the NCBI Conserved Domain Database. These tf2f domain-containing proteins include yeast $\mathrm{TAF}_{\mathrm{II}} 30 \mathrm{p}$. The prototype of this family, $\operatorname{TAF}_{\mathrm{II}} 30$, is a component of at least five yeast transcription-related complexes, NuA3, TFIID, TFIIF, SRB-mediator complex, and SWI/SNF (Henry et al. 1994; Cairns et al. 1996; Moqtaderi et al. 1996; Gustafsson et al. 1998; John et al. 2000). It is likely that all members of the tf2 family are involved in chromatin modification/remodeling processes. However, the exact role of these proteins in chromatin modification/remodeling is unclear. Deletion of $T A F_{I I} 30$ does not alter the structural integrity or the catalytic activity of the SWI/ SNF complex (Cairns et al. 1996). Similarly, the deletion of $T_{A I} 30$ results in modest decreases in NuA3 HAT activity and does not significantly alter the size of NuA3 (John et al. 2000). The Sas5p tf2f domain is similar to that in the human GAS41, ENL, and AF-9. GAS41 has been found to be amplified in low-grade gliomas (Fischer et al. 1997). Recently, GAS41 was also identified as a binding partner of NuMA, a component of the nuclear matrix in interphase cells (Harborth et al. 2000). The interaction between NuMA and GAS41 may provide a link between nuclear architecture and gene expression. Human ENL and AF-9 proteins are implicated in human acute leukemia, because $E N L$ and $A F-9$ were found fused 
Osada et al.

Table 1. Yeast strains

\begin{tabular}{|c|c|c|}
\hline Strain & Genotype & Source \\
\hline W303-1a $a^{a}$ & MATaade2-1 his3-11,15 leu2-3,112 trp1-1 ura3-1 can1-100 & A. Sachs \\
\hline YJW213 & aHis3MX6:PGAL1-3HA-SAS2 & \\
\hline YJW214 & aSAS2-3HA:His $3 M X 6$ & \\
\hline YJW219 & akanMX6:PGAL1-3HA-SAS4 & \\
\hline YJW220 & akanMX6:PGAL1-3HA-SAS5 & \\
\hline YJW228 & aSAS4-13Myc:kanMX6 & \\
\hline YJW229 & aSAS5-13Myc:kanMX6 & \\
\hline YJW230 & aHis3MX6:PGAL1-3HA-SAS4-SAS4-13Myc:kanMX6 & \\
\hline YJW231 & aHis3MX6:PGAL1-3HA-SAS2 SAS5-13Myc:kanMX6 & \\
\hline YJW243 & W303-1a [pESC/URA (pS99)] & \\
\hline YJW252 & JRY4622 (a $\operatorname{sir} 1 \Delta:: L E U 2)$ & D. Rivier \\
\hline YJW253 & DRY1655 (a sas2 $2:: T R P 1)$ & D. Rivier \\
\hline YJW254 & DRY1656 (a sas4d::kan) & D. Rivier \\
\hline YJW255 & DRY1657 (a sas5s::HIS3) & D. Rivier \\
\hline YJW258 & DRY1658 (a sir1 $\Delta:: L E U 2$ sas2- $\Delta 1:: T R P 1)$ & D. Rivier \\
\hline YJW263 & DRY1663 (a sas4t::kanMX4 sas54::HIS3) & D. Rivier \\
\hline YJW265 & a $S A S 2-13 M y c: H i s 3 M X 6$ & \\
\hline YJW269 & a $S A S 2-13 M y c: H i s 3 M X 6$ sas $4 \Delta::$ kan & \\
\hline YJW270 & a $S A S 2-13 M y c: k a n M X 6$ sas5 $5:: H I S 3$ & \\
\hline YJW271 & a $S A S 2-13 M y c: T R P 1$ sas4 $4:: \operatorname{kanMX} 4$ sas $5 \Delta:: H I S 3$ & \\
\hline LPY1382 & a $\operatorname{sas} 2 \Delta:: T R P 1$ & L. Pillus \\
\hline YJW275 & LPY1382 [pESC/URA/PGAL10-6xHIS-SAS2-FLAG (pS116)] & \\
\hline YJW276 & YJW228 [pESC/URA/PGAL10-6xHIS-SAS2-FLAG (pS116)] & \\
\hline YJW278 & LPY1382 [pESC/URA/PGAL10-6xHIS-SAS2-M1-FLAG (pS117)] & \\
\hline YJW279 & LPY1382 [pESC/URA/PGAL10-6xHIS-SAS2-M2-FLAG (pS118)] & \\
\hline YJW280 & LPY1382 [pESC/URA/PGAL10-6xHIS-SAS2-M3-FLAG (pS119)] & \\
\hline YJW281 & YJW252 [pESC/URA (pS99)] & \\
\hline YJW282 & YJW258 [pESC/URA (pS99)] & \\
\hline YJW283 & YJW258 [pESC/URA/PGAL10-6xHIS-SAS2-FLAG (pS116)] & \\
\hline YJW284 & YJW258 [pESC/URA/PGAL10-6xHIS-SAS2-M1-FLAG (pS117)] & \\
\hline YJW285 & YJW258 [pESC/URA/PGAL10-6xHIS-SAS2-M2-FLAG (pS118)] & \\
\hline YJW286 & YJW258 [pESC/URA/PGAL10-6xHIS-SAS2-M3-FLAG (pS119)] & \\
\hline YJW348 & YJW252 [pRS416/CEN/URA (pS15)] & \\
\hline YJW349 & YJW252 [pRS426/2 $\mu / U R A(p S 23)]$ & \\
\hline YJW350 & YJW258 [pRS416/CEN/URA (pS15)] & \\
\hline YJW351 & 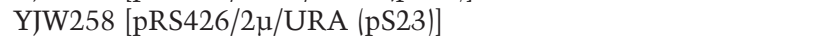 & \\
\hline YJW352 & YJW258 [pRS416/CEN/URA/PSAS2-SAS2 (pS126)] & \\
\hline YJW353 & YJW258 [pRS416/CEN/URA/PSAS2-SAS2-M1 (pS136)] & \\
\hline YJW354 & YJW258 [pRS416/CEN/URA/PSAS2-SAS2-M2 (pS137)] & \\
\hline YJW355 & YJW258 [pRS416/CEN/URA/PSAS2-SAS2-M3 (pS138)] & \\
\hline YJW356 & YJW258 [pRS426/2 $\mu /$ URA/PSAS2-SAS2 (pS127)] & \\
\hline YJW357 & 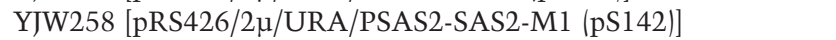 & \\
\hline YJW358 & 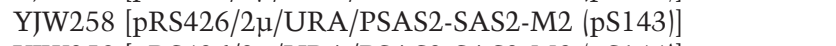 & \\
\hline YJW359 & YJW258 [pRS426/2 $\mu /$ URA/PSAS2-SAS2-M3 (pS144)] & \\
\hline YJW414 & 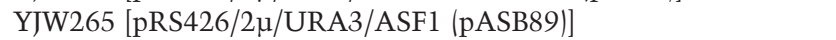 & \\
\hline YJW415 & YJW265 [pRS426/2p/URA3/ASF1-3HA (pASB90)] & \\
\hline YJW416 & YJW228 [pRS426/2p/URA3/ASF1 (pASB89)] & \\
\hline YJW417 & 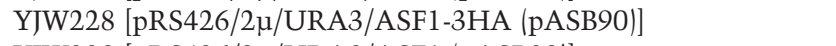 & \\
\hline YJW418 & YJW229 [pRS426/2 $\mu /$ URA3/ASF1 (pASB89)] & \\
\hline YJW419 & YJW229 [pRS426/2u/URA3/ASF1-3HA (pASB90)] & \\
\hline YJW433 & a asf $1 \Delta:: H I S 3$ & \\
\hline YJW435 & $\mathbf{a} a s f 1 \Delta:: H I S 3 \operatorname{sir} 1 \Delta:: L E U 2$ & \\
\hline YJW436 & $\mathbf{a} a s f 1 \Delta:: H I S 3$ sas $2 \Delta:: T R P 1$ & \\
\hline YJW437 & a asf1 $1 \Delta:: H I S 3$ sas $2 \Delta:: T R P 1 \operatorname{sir} 1 \Delta:: L E U 2$ & \\
\hline YJW457 & W303-1a [pRS424/2u/TRP1/PASF1-ASF1-9Myc (pASB92)] & \\
\hline YJW458 & YJW213 [pRS424/2u/TRP1/PASF1-ASF1-9Myc (pASB92)] & \\
\hline YJW459 & YJW219 [pRS424/2u/TRP1/PASF1-ASF1-9Myc (pASB92)] & \\
\hline YJW460 & YJW220 [pRS424/2u/TRP1/PASF1-ASF1-9Myc (pASB92)] & \\
\hline W303-1 $b^{b}$ & MAT $\alpha$ ade2-1 his3-11,15 leu2-3,112 trp1-1 ura3-1 can1-100 & R. Rothstein \\
\hline YGM75 & $h m r \Delta E \Delta B$ & G. Micklem \\
\hline SY560 & $h m r \Delta E \Delta B$ asf1::His5 $5^{+}$ & \\
\hline
\end{tabular}


Table 1. (Continued)

\begin{tabular}{|c|c|c|}
\hline Strain & Genotype & Source \\
\hline YAB53 & $h m r \Delta A \Delta E$ & A. Brand \\
\hline SY557 & hmr $\Delta A \Delta E$ asf1::His5 ${ }^{+}$ & \\
\hline YAB197 & $h m r \Delta B$ sir1::LEU2 & A. Brandt \\
\hline SY558 & hmr $\Delta B$ sir1::LEU2 asf1::His5 ${ }^{+}$ & \\
\hline YJW251 ${ }^{c}$ & JRY2728 MATa his4 & D. Rivier \\
\hline $\mathrm{AY} 1281^{c}$ & MATa his1 & K. Arndt \\
\hline $\mathrm{AY} 1283^{c}$ & $M A T \alpha$ his1 & K. Arndt \\
\hline $\mathrm{YJW} 287^{d}$ & 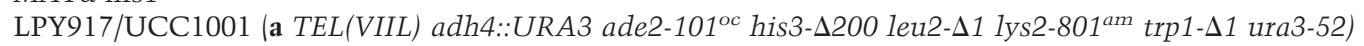 & L. Pillus \\
\hline YJW288 & LPY1285 a sir1s::HIS3 & L. Pillus \\
\hline YJW289 & LPY2058 a sas2L::TRP1 & L. Pillus \\
\hline YJW290 & LPY2062 a sas $2 \Delta:: T R P 1 \operatorname{sir} 1 \Delta:: H I S 3$ & L. Pillus \\
\hline YJW324 & YJW287 [pRS425/2u/LEU (pS20)] & \\
\hline YJW326 & 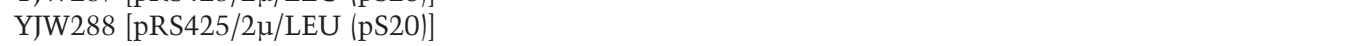 & \\
\hline YJW328 & YJW289 [pRS425/2u/LEU (pS20)] & \\
\hline YJW330 & YJW289 [pRS425/2u/LEU/PSAS2-SAS2 (pS123)] & \\
\hline YJW332 & YJW290 [pRS425/2p/LEU (pS20)] & \\
\hline YJW334 & YJW290 [pRS425/2u/LEU/PSAS2-SAS2 (pS123)] & \\
\hline YJW369 & YJW289 [pRS425/2u/LEU/PSAS2-SAS2-M1 (pS139)] & \\
\hline YJW370 & 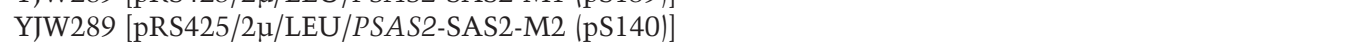 & \\
\hline YJW371 & 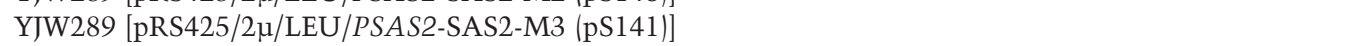 & \\
\hline YJW375 & YJW290 [pRS425/2u/LEU/PSAS2-SAS2-M1 (pS139)] & \\
\hline YJW376 & YJW290 [pRS425/2u/LEU/PSAS2-SAS2-M2 (pS140)] & \\
\hline YJW377 & YJW290 [pRS425/2u/LEU/PSAS2-SAS2-Me (pS141)] & \\
\hline
\end{tabular}

\footnotetext{
${ }^{a}$ Strains below are isogenic with W303-1a.

${ }^{b}$ Strains below are isogenic with W303-1b.

${ }^{c}$ YJW251, AY1281, and AY1283 are lawn tester strains for mating assays.

${ }^{d}$ Strains below are isogenic with YJW287.
}

to the $A L L-1$ gene in leukemias with translocations (Tkachuk et al. 1992; Negrini et al. 1993). Further analysis of the function of Sas5p in Sas $2 p$-mediated silencing might help us understand how human tf2f proteins such as ENL, AF-9, and GAS41 contribute to tumorigenesis in humans.

\section{ASF1 and SAS2 enhance the silencing defect at HML in a sir1 mutant}

We showed that chromatin assembly factor Asflp interacts with components of the SAS complex in vivo. Individually, asf1 mutants do not show significant derepression of silencing at mating-type loci (Le et al. 1997; Singer et al. 1998; Tyler et al. 1999), but the double asf1 sir1 deletion strain displayed a much more severe silencing defect at $H M L$ than did deletion of either gene alone. This phenotype of asf1 is similar to that which is observed for sas genes on silencing at $H M L$ (Xu et al. 1999b). Furthermore, asf1 mutations, like sas mutations, restored silencing at mutated $H M R$ silencers. These genetic results suggest that $A S F 1$ and $S A S 2 / 4 / 5$ function in the same pathway to regulate silencing at $H M L$ and $H M R$. Although asf1, sas2, sas4, and sas 5 mutants have very similar silencing phenotypes at $H M L$ and $H M R$, they have drastically different properties with respect to telomeric silencing. Although asf1 mutants show little or no telomeric silencing defect (Singer et al. 1998), sas2, sas4, and sas 5 mutants are totally defective in telomeric silencing (Reifsnyder et al. 1996).
Nucleosome assembly may be important to generate the silent state, because deletion of CAF-1 subunits leads to a significant decrease in silencing at telomeres and a subtle weakening of silencing at $H M L$ and $H M R$ (Enomoto and Berman 1998). CAC1 encodes the largest subunit of CAF-1 that assembles newly synthesized histones onto replicated DNA. A cac1 mutation enhanced the mating defect of a sir1 deletion strain. CAF-1 contributes to the maintenance, but not the re-establishment, of silencing at the mating loci (Enomoto and Berman 1998). Mutations in the genes for either chromatin assembly factor, CAF-1 or Asf1p, enhance the silencing defect of sir1 mutants. ASF1 function also might be involved in the maintenance of silent mating type genes. Because ASF1 and SAS2/4/5 genetically function in the same pathway to repress the $H M L$ locus, the interaction between the SAS complex and Asflp might have a role for maintenance.

\section{Materials and methods}

\section{Yeast strains and manipulations}

Strains used in this study are described in Table 1. All of the chromosomal integrated tagged-strains were generated by the one-step PCR-mediated technique as described elsewhere (Longtine et al. 1998). The template plasmids were kindly provided by Dr. Peter Philippsen (Institut für Angewandte Microbiologie, Biozentrum, Universität Basel, Switzerland). A DNA fragment used to replace the ASF1 open reading frame (ORF) with the $S$. pombe his $5^{+}$gene was synthesized by PCR using 
synthetic oligonucleotides and the plasmid pME3 as described (Wach et al. 1997). Standard yeast manipulations were performed as described (Guthrie and Fink 1991).

\section{Plasmid construction}

The C-terminal Flag-tagged Sas2p, Sas4p, and Sas5p expression vectors, pS100, pS120, and pS104, were constructed by fusing the SAS2, SAS4, and SAS5 ORFs, which were amplified by PCR, and the products were subcloned into the SpeI site of the Flagtagged protein expression vector pESC/URA (pS99; Stratagene). For the $\mathrm{N}$-terminal $6 \mathrm{xHis}$-tagged and the C-terminal Flag-tagged Sas $2 p$, Sas $4 p$, and Sas $5 p$ expression vectors pS116, pS128, and pS129, the double-stranded oligonucleotide containing 6xHistagged sequence was ligated into the NotI site of pS100, pS120, and pS104. Plasmids expressing Sas $2 p$ regulated by the endogenous promoter were generated by PCR amplification of the SAS2-coding sequences along with $1 \mathrm{~kb}$ upstream region sequence from yeast genomic DNA (pS123, pS126, pS127). The amplified product was cloned into pRS416 (pS15), pRS425 (pS20), and pRS426 (pS23). All fragments generated by PCR were verified by sequencing. Mutations within the putative acetylCoA binding site of SAS2 (pS117-119, pS136-pS144) were introduced using the QuickChange site-directed mutagenesis kit (Stratagene) following manufacturer's protocols, and mutations were confirmed by sequencing. ASB90 (ASF1-[HA $]_{3}$ in pRS426) was created as follows. First, PCR mutagenesis was used to replace the stop codon at the $3^{\prime}$ end of the ASF1 ORF in pLS67 (ASF1 in pUC18) with a NotI site to create pASB31. A fragment encoding a triple repeat of the HA epitope flanked by Not 1 restriction sites was inserted into the Not1 site of pASB31 to create pASB35. An XbaI-EcoRI fragment encoding the C terminus of Asf1p in plasmid pLS27 (full length ASF1 in YCp50) was replaced with an $X b a \mathrm{I}-E c o$ RI fragment from pASB35 to create pASB41 (ASF1-[HA $]_{3}$ in YCp50). A SalI-EcoRI fragment from pASB41 containing ASF1-[HA $]_{3}$ was cloned into pRS426 to create ASB90. ASB92 (ASF1-[Myc $\left.]_{9}\right)$ was created as follows. A Not 1 fragment containing 13 tandem copies of the Myc epitope was inserted into the Not1 site of ASB31 so that the Myc tag was in-frame with the C terminus of Asflp. A Nde1 fragment that encodes the Myc-tagged C terminus of Asflp was then used to replace the sequences for the untagged $\mathrm{C}$ terminus of $A S F 1$ in pRS424. ASB89 (ASF1 in pRS426) was created by cloning a BamHI-HindIII fragment containing ASF1 from pLS27 into pRS426. ASB92 ASF1[Myc $]_{9}$ in pRS424. To create the GST-Asflp fusion protein expression vector (pS173), the ORF of ASF1 gene was subcloned into the BamHI and EcoRI sites of the glutathione $S$-transferase (GST) expression vector pGEX-2T (AmershamPharmacia).

\section{Purification of the SAS complex}

Whole cell extracts were prepared essentially as a previously published procedure (Eberharter et al. 1998). Approximately 4 $\mathrm{mg}$ and $0.4 \mathrm{mg}$ of whole cell extracts were loaded onto a $24-\mathrm{mL}$ Superose 6 HR 10/30 and a 2.4-mL Superdex 200 PC 3.2/30 column (Amersham-Pharmacia), respectively, equilibrated in buffer C (40 mM HEPES at pH 7.8, $500 \mathrm{mM} \mathrm{NaCl}, 10 \%$ glycerol, $0.1 \%$ Tween $20,1 \mathrm{mM}$ phenylmethylsulfonyl fluoride, $0.5 \mu \mathrm{g} /$ $\mathrm{mL}$ leupeptin, and $0.5 \mu \mathrm{g} / \mathrm{mL}$ pepstatin A). For Figure 1, whole cell extracts from two liters of SAS2-HA strain (YJW214) were mixed with $1.6 \mathrm{~mL}$ of $\mathrm{Ni}^{2+}$-NTA agarose (QIAGEN) overnight. The unbound fraction was loaded onto a 20-mL MonoQ HR 16/10 column (Amersham-Pharmacia). The bound fraction was eluted in a 25-column-volume gradient from $0.1 \mathrm{M}$ to $0.5 \mathrm{M}$ $\mathrm{NaCl}$ in buffer B (50 mM Tris at $\mathrm{pH} 8.0,10 \%$ glycerol, $0.1 \%$
Tween 20, and protease inhibitors described above). Fractions containing Sas2--HA were concentrated over a 1-mL MonoQ HR 5/5 column (Amersham-Pharmacia) using the same gradient as described above. The Sas2-HA fractions were subjected to gel filtration by a Superose 6 . The fractions were precipitated with TCA before gel electrophoresis. All gel filtration columns were calibrated using a high-molecular-weight filtration calibration kit (Amersham-Pharmacia).

For mass spectrometry analyses, whole cell extracts were prepared from $12 \mathrm{~L}$ of yeast strain YJW276. The extracts were bound to $10 \mathrm{~mL}$ of $\mathrm{Ni}^{2+}$-NTA agarose resin overnight. Then resin was washed with the extraction buffer $(40 \mathrm{mM}$ HEPES at pH 7.5, $350 \mathrm{mM} \mathrm{NaCl}, 10 \%$ glycerol, $0.1 \%$ Tween-20, and protease inhibitors) and $20 \mathrm{mM}$ imidazole solution containing 100 $\mathrm{mM} \mathrm{NaCl}, 10 \%$ glycerol, $0.1 \%$ Tween-20, and protease inhibitors described above. Bound proteins were eluted with $30 \mathrm{~mL}$ of $300 \mathrm{mM}$ imidazole solution. The imidazole eluate was loaded onto a 1-mL MonoQ column. Retained proteins were eluted in a 10-column-volume gradient from $0.1 \mathrm{M}$ to $0.8 \mathrm{M} \mathrm{NaCl}$ in buffer B. Dithiothreitol (DTT) and anti-Flag M2 affinity resin was added to the fraction containing Sas $4 \mathrm{p}-\mathrm{Myc}$. Beads were washed extensively with buffer B containing $0.5 \mathrm{M} \mathrm{NaCl}$ and 1 $\mathrm{mM}$ DTT, and bound proteins were eluted with buffer B containing $0.5 \mathrm{M} \mathrm{NaCl}, 1 \mathrm{mM}$ DTT and $0.7 \mathrm{mg} / \mathrm{mL}$ Flag peptide (Sigma). The eluate was subjected to a $2.4-\mathrm{mL}$ Superdex 200 PC $3.2 / 30$ gel filtration column equilibrated in the buffer $\mathrm{C}$.

\section{Western blotting and antibodies}

Ten microliters of each fraction were electrophoresed on a SDSPAGE gel, transferred to nitrocellulose, and detected by ECL Western blotting analysis detection system (Amersham-Pharmacia). Anti-Myc (clone 9E10, Boehringer), horseradish peroxidase-conjugated anti-HA (clone 3F10, Boehringer), and anti-Flag M2 (Sigma) antibodies were used.

\section{Immunoprecipitations and GST pull-down assays}

One microliter of the anti-HA antibody (clone 16B12, Covance) was mixed with $400 \mu \mathrm{g}$ of whole cell extracts or $72 \mu \mathrm{L}$ of Superose 6 fractions that were diluted to appropriate salt concentration of $150 \mathrm{mM} \mathrm{NaCl}$ with buffer and incubated on ice for $8 \mathrm{~h}$. Twenty microliters of protein $\mathrm{G}$-Sepharose beads were added to the sample and incubated at $4^{\circ} \mathrm{C}$ for $8 \mathrm{~h}$. In some experiments, ethidium bromide (EtBr) was added $(50 \mu \mathrm{g} / \mathrm{mL})$, and the extracts were incubated on ice for $30 \mathrm{~min}$. Precipitates were removed by centrifugation at $4^{\circ} \mathrm{C}$ for $10 \mathrm{~min}$, and the supernatant was used for immunoprecipitation experiments. For micrococcal nuclease (MNase) treatment, extracts were treated with 0.4 units of MNase, $0.5 \mathrm{mM} \mathrm{CaCl}_{2}$, and $0.5 \mathrm{mM} \mathrm{MgCl}_{2}$ at $27^{\circ} \mathrm{C}$ for $10 \mathrm{~min}$. Beads were washed with extraction buffer or Buffer $\mathrm{C}$ containing $150 \mathrm{mM} \mathrm{NaCl}$ before gel electrophoresis.

GST fusion proteins were expressed in Escherichia coli as described by the manufacturer (Amersham-Pharmacia) and cross-linked to glutathione sepharose 4B with dimethylpimelimidate (Harlow and Lane 1988). The N-terminal 6xHis-tagged and the C-terminal Flag-tagged Sas2, Sas4, and Sas5 proteins were individually expressed from pS116, pS128, and pS129 with T3 polymerase using TNT-coupled reticulocyte lysate system (Promega). Five microliters of the reticulocyte lysate reaction containing $\left[{ }^{35} \mathrm{~S}\right]$ methionine-labeled Sas proteins were incubated in buffer $\mathrm{B}$ containing $350 \mathrm{mM} \mathrm{NaCl}$ at $4^{\circ} \mathrm{C}$ overnight with GST-fusion proteins cross-linked to glutathione Sepharose 4B. Beads were washed with buffer B containing $350 \mathrm{mM} \mathrm{NaCl}$ before gel electrophoresis. 


\section{Mass spectrometry}

The Sas 2 complex fraction was electrophoresed through a $4 \%$ to $15 \%$ SDS-polyacrylamide gradient gel. Silver-stained protein bands were excised and digested in gel trypsin. Proteins were identified by microcolumn high-performance liquid chromatography electrospray ionization tandem mass spectrometry and database searching (Grant et al. 1998).

\section{Mating and telomeric silencing assays}

Mating assays were performed as described previously (Sprague 1991). The patches from the strains containing pRS416- and pRS426-based plasmids were replica plated to a lawn of $\alpha$-cells (AY1283) grown on dextrose plates. Galactose plates were used for mating assays with the strains carrying the pESC plasmids. Quantitative mating assay was performed as described previously (Ehrenhofer-Murray et al. 1997). Serial dilutions of test strains were mixed with $1.0 \times 10^{7}$ cells of a MAT $\alpha$ lawn (AY1283 or YJW251) or a MATa lawn (AY1281) and plated onto selective medium and rich medium to determine the number of viable cells. For the experiments in Figure 5, mating efficiency was expressed as a percentage of diploid cells formed per viable cells. For the experiments in Figure 7, mating efficiency was expressed as the number of diploids formed per viable cells and was normalized to the efficiency of an isogenic wild-type strain. Mating efficiencies are the average of three independent experiments. For the telomeric silencing assay, three microliters of 10 -fold serial dilutions of the saturated cultures were spotted on plates lacking leucine (for plasmid selection) and 5-FOA containing plates.

\section{Acknowledgments}

We thank members of the Workman, Simpson, and Reese Laboratories at Penn State for valuable discussions. We are also grateful to Peter Philippsen for plasmids, Lorraine Pillus for providing the LPY strains, and David $\mathrm{H}$. Rivier for a generous gift of DRY strains. We thank Song Tan for structure analysis of the HAT domain mutants, Jean Bucaria for excellent technical assistance, and Thomas Kusch for useful advice and critical reading of the manuscript. We also thank Tsutomu Nishihara for encouragement of this work. Support for this work was provided by grants from NIGMS to J.L.W and to R.S., and NCRR Yeast Center Grant RR11823 to J.Y.R. C.E.B. is a Leukemia and Lymphoma Society Postdoctoral Fellow. J.L.W. is an Associate Investigator of the HHMI.

The publication costs of this article were defrayed in part by payment of page charges. This article must therefore be hereby marked "advertisement" in accordance with 18 USC section 1734 solely to indicate this fact.

\section{References}

Allard, S., Utley, R.T., Savard, J., Clarke, A., Grant, P., Brandl, C.J., Pillus, L., Workman, J.L., and Côté, J. 1999. NuA4, an essential transcription adaptor/histone $\mathrm{H} 4$ acetyltransferase complex containing Esalp and the ATM-related cofactor Tralp. EMBO J. 18: 5108-5119.

Altschul, S.F., Madden, T.L., Schaffer, A.A., Zhang, J., Zhang, Z., Miller, W., and Lipman, D.J. 1997. Gapped BLAST and PSI-BLAST: A new generation of protein database search programs. Nucleic Acids Res. 25: 3389-3402.

Aparicio, O.M., Billington, B.L., and Gottschling, D.E. 1991. Modifiers of position effect are shared between telomeric and silent mating-type loci in S. cerevisiae. Cell 66: 12791287.

Borrow, J., Stanton, Jr., V.P., Andresen, J.M., Becher, R., Behm, F.G., Chaganti, R.S., Civin, C.I., Disteche, C., Dube, I., Frischauf, A.M., et al. 1996. The translocation $\mathrm{t}(8 ; 16)(\mathrm{p} 11 ; \mathrm{p} 13)$ of acute myeloid leukaemia fuses a putative acetyltransferase to the CREB-binding protein. Nat. Genet. 14: 33-41.

Brown, C.E., Lechner, T., Howe, L., and Workman, J.L. 2000. The many HATs of transcription coactivators. Trends Biochem. Sci. 25: 15-19.

Cairns, B.R., Henry, N.L., and Kornberg, R.D. 1996. TFG/ TAF30/ANC1, a component of the yeast SWI/SNF complex that is similar to the leukemogenic proteins ENL and AF-9. Mol. Cell. Biol. 16: 3308-3316.

Champagne, N., Bertos, N.R., Pelletier, N., Wang, A.H., Vezmar, M., Yang, Y., Heng, H.H., and Yang, X.J. 1999. Identification of a human histone acetyltransferase related to monocytic leukemia zinc finger protein. I. Biol. Chem. 274: 28528-28536.

Cockell, M., Gotta, M., Palladino, F., Martin, S.G., and Gasser, S.M. 1998. Targeting Sir proteins to sites of action: A general mechanism for regulated repression. Cold Spring Harb. Symp. Quant. Biol. 63: 401-412.

Creaven, M., Hans, F., Mutskov, V., Col, E., Caron, C., Dimitrov, S., and Khochbin, S. 1999. Control of the histone-acetyltransferase activity of Tip60 by the HIV-1 transactivator protein, Tat. Biochemistry 38: 8826-8830.

Dutta, A. and Bell, S.P. 1997. Initiation of DNA replication in eukaryotic cells. Annu. Rev. Cell Dev. Biol. 13: 293-332.

Eberharter, A., John, S., Grant, P.A., Utley, R.T., and Workman, J.L. 1998. Identification and analysis of yeast nucleosomal histone acetyltransferase complexes. Methods 15: 315-321.

Ehrenhofer-Murray, A.E., Rivier, D.H., and Rine, J. 1997. The role of Sas2, an acetyltransferase homologue of Saccharomyces cerevisiae, in silencing and ORC function. Genetics 145: 923-934.

Eisen, A., Utley, R.T., Nourani, A., Allard, S., Schmidt, P., Lane, W.S., Lucchesi, J.C., and Côté, J. 2000. The yeast NuA4 and Drosophila MSL complexes contain homologous subunits important for transcriptional regulation. J. Biol. Chem. 276: 3484-3491.

Emili, A., Schieltz, D.M., Yates, J.R., and Hartwell, L.H. 2001. Dynamic interaction of DNA damage checkpoint protein Rad53 with chromatin assembly factor Asf1. Mol. Cell 7: 1320.

Enomoto, S. and Berman, J. 1998. Chromatin assembly factor I contributes to the maintenance, but not the re-establishment, of silencing at the yeast silent mating loci. Genes \& Dev. 12: 219-232.

Fischer, U., Heckel, D., Michel, A., Janka, M., Hulsebos, T., and Meese, E. 1997. Cloning of a novel transcription factor-like gene amplified in human glioma including astrocytoma grade I. Hum. Mol. Genet. 6: 1817-1822.

Galarneau, L., Nourani, A., Boudreault, A.A., Zhang, Y., Heliot, L., Allard, S., Savard, J., Lane, W.S., Stillman, D.J., and Côté, J. 2000. Multiple links between the NuA4 histone acetyltransferase complex and epigenetic control of transcription. Mol. Cell 5: 927-937.

Grant, P.A., Schieltz, D., Pray-Grant, M.G., Steger, D.J., Reese, J.C., Yates III, J.R., and Workman, J.L. 1998. A subset of TAF(II)s are integral components of the SAGA complex required for nucleosome acetylation and transcriptional stimulation. Cell 94: 45-53.

Gustafsson, C.M., Myers, L.C., Beve, J., Spahr, H., Lui, M., Erdjument-Bromage, H., Tempst, P., and Kornberg, R.D. 1998. Identification of new mediator subunits in the RNA poly- 
merase II holoenzyme from Saccharomyces cerevisiae. I. Biol. Chem. 273: 30851-30854.

Guthrie, C. and Fink, G.R. 1991. Guide to yeast genetics and molecular biology. Academic Press, San Diego, CA.

Harborth, J., Weber, K., and Osborn, M. 2000. GAS41, a highly conserved protein in eukaryotic nuclei, binds to NuMA. I. Biol. Chem. 275: 31979-31985.

Harlow, E. and Lane, D. 1988. Antibodies: A laboratory manual. Cold Spring Harbor Laboratory Press, Cold Spring Harbor, NY.

Henry, N.L., Campbell, A.M., Feaver, W.J., Poon, D., Weil, P.A., and Kornberg, R.D. 1994. TFIIF-TAF-RNA polymerase II connection. Genes \& Dev. 8: 2868-2878.

Howe, L., Brown, C.E., Lechner, T., and Workman, J.L. 1999. Histone acetyltransferase complexes and their link to transcription. Crit. Rev. Eukaryot. Gene Expr. 9: 231-243.

Hu, F., Alcasabas, A.A., and Elledge, S.J. 2001. Asf1 links Rad53 to control of chromatin assembly. Genes \& Dev. 15: 10611066.

Iizuka, M. and Stillman, B. 1999. Histone acetyltransferase $\mathrm{HBO} 1$ interacts with the ORC1 subunit of the human initiator protein. J. Biol. Chem. 274: 23027-23034.

Ikura, T., Ogryzko, V.V., Grigoriev, M., Groisman, R., Wang, J., Horikoshi, M., Scully, R., Qin, J., and Nakatani, Y. 2000. Involvement of the TIP60 histone acetylase complex in DNA repair and apoptosis. Cell 102: 463-473.

John, S., Howe, L., Tafrov, S.T., Grant, P.A., Sternglanz, R., and Workman, J.L. 2000. The something about silencing protein,

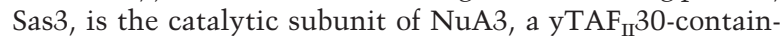
ing HAT complex that interacts with the Spt16 subunit of the yeast CP (Cdc68/Pob3)-FACT complex. Genes \& Dev. 14: 1196-1208.

Kelly, T.J., Qin, S., Gottschling, D.E., and Parthun, M.R. 2000. Type B histone acetyltransferase Hatlp participates in telomeric silencing. Mol. Cell. Biol. 20: 7051-7058.

Le, S., Davis, C., Konopka, J.B., and Sternglanz, R. 1997. Two new S-phase-specific genes from Saccharomyces cerevisiae. Yeast 13: 1029-1042.

Longtine, M.S., McKenzie III, A., Demarini, D.J., Shah, N.G., Wach, A., Brachat, A., Philippsen, P., and Pringle, J.R. 1998. Additional modules for versatile and economical PCR-based gene deletion and modification in Saccharomyces cerevisiae. Yeast 14: 953-961.

Lustig, A.J. 1998. Mechanisms of silencing in Saccharomyces cerevisiae. Curr. Opin. Genet. Dev. 8: 233-239.

Moqtaderi, Z., Yale, J.D., Struhl, K., and Buratowski, S. 1996. Yeast homologues of higher eukaryotic TFIID subunits. Proc. Natl. Acad. Sci. 93: 14654-14658.

Mullen, J.R., Kayne, P.S., Moerschell, R.P., Tsunasawa, S., Gribskov, M., Colavito-Shepanski, M., Grunstein, M., Sherman, F., and Sternglanz, R. 1989. Identification and characterization of genes and mutants for an $\mathrm{N}$-terminal acetyltransferase from yeast. EMBO J. 8: 2067-2075.

Munakata, T., Adachi, N., Yokoyama, N., Kuzuhara, T., and Horikoshi, M. 2000. A human homologue of yeast anti-silencing factor has histone chaperone activity. Genes Cells 5: 221-233.

Negrini, M., Felix, C.A., Martin, C., Lange, B.J., Nakamura, T., Canaani, E., and Croce, C.M. 1993. Potential topoisomerase II DNA-binding sites at the breakpoints of a $t(9 ; 11)$ chromosome translocation in acute myeloid leukemia. Cancer Res. 53: 4489-4492.

Pillus, L. and Rine, J. 1989. Epigenetic inheritance of transcriptional states in S. cerevisiae. Cell 59: 637-647.

Reifsnyder, C., Lowell, J., Clarke, A., and Pillus, L. 1996. Yeast SAS silencing genes and human genes associated with AML and HIV-1 Tat interactions are homologous with acetyltransferases. Nat. Genet. 14: 42-49.

Sharma, M., Zarnegar, M., Li, X., Lim, B., and Sun, Z. 2000. Androgen receptor interacts with a novel MYST protein, HBO1. J. Biol. Chem. 275: 35200-35208.

Sherman, J.M. and Pillus, L. 1997. An uncertain silence. Trends Genet. 13: 308-313.

Singer, M.S., Kahana, A., Wolf, A.J., Meisinger, L.L., Peterson, S.E., Goggin, C., Mahowald, M., and Gottschling, D.E. 1998. Identification of high-copy disruptors of telomeric silencing in Saccharomyces cerevisiae. Genetics 150: 613-632.

Smith, E.R., Eisen, A., Gu, W., Sattah, M., Pannuti, A., Zhou, J., Cook, R.G., Lucchesi, J.C. and Allis, C.D. 1998. ESA1 is a histone acetyltransferase that is essential for growth in yeast. Proc. Natl. Acad. Sci. 95: 3561-3565.

Smith, E.R., Pannuti, A., Gu, W., Steurnagel, A., Cook, R.G., Allis, C.D., and Lucchesi, J.C. 2000. The Drosophila MSL complex acetylates histone $\mathrm{H} 4$ at lysine 16, a chromatin modification linked to dosage compensation. Mol. Cell. Biol. 20: 312-318.

Sprague, Jr., G.F. 1991. Assay of yeast mating reaction. Methods Enzymol. 194: 77-93.

Sterner, D.E. and Berger, S.L. 2000. Acetylation of histones and transcription-related factors. Microbiol. Mol. Biol. Rev. 64: 435-459.

Sun, Z.W. and Hampsey, M. 1999. A general requirement for the Sin3-Rpd3 histone deacetylase complex in regulating silencing in Saccharomyces cerevisiae. Genetics 152: 921-932.

Sutton, A., Bucaria, J., Osley, M.A., and Sternglanz, R. 2001. Yeast ASF1 protein is required for cell-cycle regulation of histone gene transcription. Genetics 158: 587-596.

Takechi, S. and Nakayama, T. 1999. Sas3 is a histone acetyltransferase and requires a zinc finger motif. Biochem. Biophys. Res. Commun. 266: 405-410.

Tkachuk, D.C., Kohler, S., and Cleary, M.L. 1992. Involvement of a homolog of Drosophila trithorax by 11q23 chromosomal translocations in acute leukemias. Cell 71: 691-700.

Tyler, J.K., Adams, C.R., Chen, S.R., Kobayashi, R., Kamakaka, R.T., and Kadonaga, J.T. 1999. The RCAF complex mediates chromatin assembly during DNA replication and repair. $\mathrm{Na}$ ture 402: 555-560.

Wach, A., Brachat, A., Alberti-Segui, C., Rebischung, C., and Philippsen, P. 1997. Heterologous HIS3 marker and GFP reporter modules for PCR-targeting in Saccharomyces cerevisiae. Yeast 13: 1065-1075.

Xu, E.Y., Kim, S., Replogle, K., Rine, J., and Rivier, D.H. 1999a. Identification of SAS4 and SAS5, two genes that regulate silencing in Saccharomyces cerevisiae. Genetics 153: 13-23.

Xu, E.Y., Kim, S., and Rivier, D.H. 1999b. SAS4 and SAS5 are locus-specific regulators of silencing in Saccharomyces cerevisiae. Genetics 153: 25-33.

Yamamoto, T. and Horikoshi, M. 1997. Novel substrate specificity of the histone acetyltransferase activity of HIV-1-Tat interactive protein Tip60. J. Biol. Chem. 272: 30595-30598.

Yan, Y., Barlev, N.A., Haley, R.H., Berger, S.L., and Marmorstein, R. 2000. Crystal structure of yeast esal suggests a unified mechanism for catalysis and substrate binding by histone acetyltransferases. Mol. Cell 6: 1195-1205. 


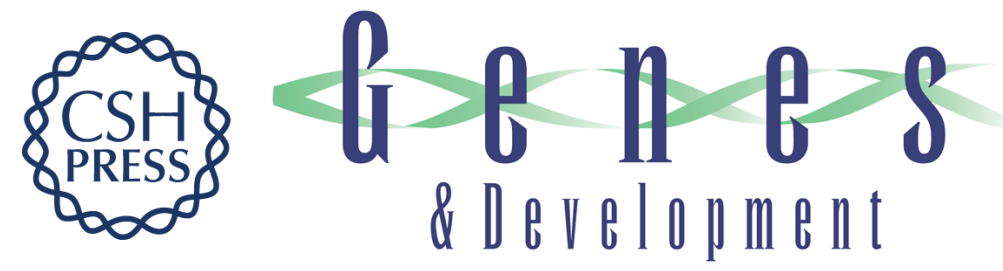

\section{The yeast SAS (something about silencing) protein complex contains a MYST-type putative acetyltransferase and functions with chromatin assembly factor ASF1}

Shigehiro Osada, Ann Sutton, Nemone Muster, et al.

Genes Dev. 2001, 15:

Access the most recent version at doi:10.1101/gad.907201

\section{References This article cites 53 articles, 25 of which can be accessed free at: http://genesdev.cshlp.org/content/15/23/3155.full.html\#ref-list-1}

\section{License}

Email Alerting

Service

Receive free email alerts when new articles cite this article - sign up in the box at the top right corner of the article or click here.

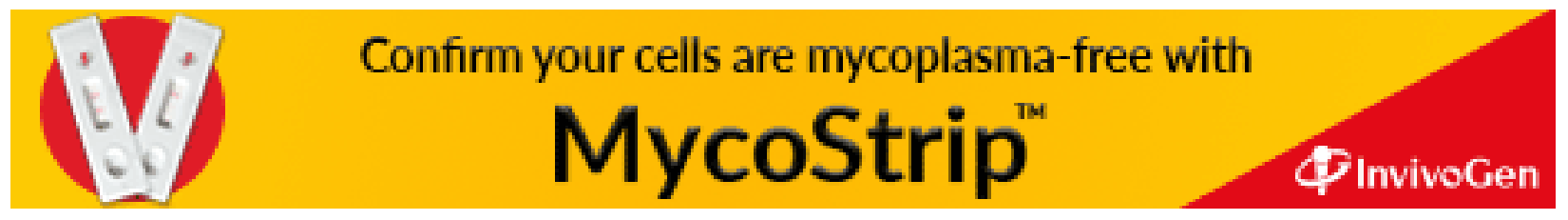

